\title{
PAPEL DE LA UNIDAD DE CUIDADOS INTENSIVOS PEDIÁTRICOS EN LA EVALUACIÓN Y SOPORTE DEL PACIENTE CON PATOLOGÍA DE LA VÍA AÉREA. ¿CÓMO LO HACEMOS?
}

\section{Role of the pediatric intensive care unit in the evaluation and support of the patient with pathology of the airway. How do we do it?}

\author{
Pedro Pablo OYAGÜEZ-UGIDOS'; Isabel BLANCO-GÓMEZ1; María GARCíA-GONZÁLEZ1; \\ María Luz ALONSO-ÁLVAREZ²
}

Hospital Universitario de Burgos. ${ }^{1}$ Unidad de Cuidados Intensivos Pediátricos. Servicio de Pediatría. ${ }^{2}$ Unidad de Trastornos durante el Sueño. Burgos. España.

Correspondencia: poyaguez@saludcastillayleon.es

Fecha de recepción: 21 de marzo de 2017

Fecha de aceptación: 7 de mayo de 2017

Fecha de publicación: 10 de mayo de 2017

Fecha de publicación del fascículo: 1 de marzo de 2018

Conflicto de intereses: Los autores declaran no tener conflictos de intereses

Imágenes: Los autores declaran haber obtenido las imágenes con el permiso de los pacientes

Política de derechos y autoarchivo: se permite el autoarchivo de la versión post-print (SHERPA/RoMEO)

Licencia CC BY-NC-ND. Licencia Creative Commons Atribución-NoComercial-SinDerivar 4.0 Internacional

Universidad de Salamanca. Su comercialización está sujeta al permiso del editor

RESUMEN

Introducción y Objetivo: La patología de vía aérea comprende un amplio espectro de manifestaciones clínicas que incluyen distintos grados de insuficiencia respiratoria y/o trastornos respiratorios durante el sueño. El manejo se realiza de forma multidisciplinar, colaborando con ORL, Cirugía Infantil y Unidad de trastornos respiratorios del sueño. La ventilación no invasiva (VNI) ha mostrado su utilidad terapéutica en el paciente pediátrico, tanto en situaciones agudas como crónicas. La indicación más frecuente es el fallo respiratorio agudo. Aunque no existen numerosas contraindicaciones para su aplicación deben conocerse para evitar los fallos de esta técnica. Material y método: Presentamos una revisión de los conceptos básicos, indicaciones, contraindicaciones y nuestra metodología de aplicación de VNI en pacientes pediátricos. Conclusiones: Un porcentaje importante de los niños ingresados en UCIP precisan ventilación mecánica, siendo la insuficiencia respiratoria aguda la indicación más frecuente. En situaciones agudas, se aplica VNI para estabilizar al paciente, mientras que en casos crónicos puede ser útil durante la fase de estudio. Puede precisarse como apoyo durante el estudio fibroscópico, y para titular los parámetros de VNI en pacientes subsidiarios de soporte domiciliario.

PALABRAS CLAVE ventilación no invasiva; insuficiencia respiratoria aguda; insuficiencia respiratoria crónica; interfaces; cuidados intensivos pediátricos tations that include different degrees of respiratory failure and / or respiratory disorders during 
sleep. The management is done in a multidisciplinary way, collaborating with ENT, Pediatric surgeon and Sleep Respiratory Disorders Unit. Noninvasive Ventilation (NIV) has shown its therapeutic utility in the pediatric patient, in both acute and chronic situations. The most frequent indication is acute respiratory failure. Although there are not many contraindications for its application should be known to avoid failures of this technique. Material and method: We present a review of basic concepts, indications, contraindications and our methodology of NIV in pediatric patients. Conclusions: A significant percentage of children admitted to PICU require mechanicaln ventilation. The most frequent indication is respiratory failure. In acute situations, NIV is applied to stabilize the patient, while in chronic cases can be useful during the study phase. It can be specified as support during the Fibroscopic study, and to titrate NIV parameters in supportive patients domiciliary.

KEYWORDS non-invasive ventilation; acute respiratory failure; chronic respiratory failure; interfaces; pediatric intensive care

\section{INTRODUCCIÓN}

La patología de vía aérea comprende un amplio espectro de manifestaciones clínicas que incluyen distintos grados de insuficiencia respiratoria y/o trastornos respiratorios durante el sueño. Tanto para el estudio como en la estabilización de casos con insuficiencia respiratoria, la ventilación no invasiva (VNI) es una herramienta a disposición del clínico. En este artículo revisamos el estado actual del tema y presentamos datos de su aplicación en la unidad de cuidados intensivos pediátricos (UCIP) del Complejo Asistencial de Burgos. Es importante conocer las características de la respiración en el niño, sus peculiaridades anatómicas y fisiológicas, y los mecanismos de respuesta en situaciones de patología. Sin entrar a profundizar en cada aspecto, recordemos que hay dos puntos críticos en la vía aérea: a nivel faríngeo (por la hipertrofia amigdalar y la colapsabilidad) y a nivel del cricoides (zona de menor calibre de la vía aérea superior). Por otra parte, la elasticidad de la parrilla costal, su horizontalización en el lactante y niño pequeño, y la disposición diafragmática, condicionan una menor eficiencia de la musculatura respiratoria, y una propensión a la respiración paradójica en situaciones de esfuerzo respiratorio. En este contexto, hemos de realizar una adecuada valoración, que permita establecer la estrategia terapéutica más apropiada. Al igual que en el adulto [1-3], la ventilación no invasiva (VNI) ha mostrado su utilidad en el paciente pediátrico [4], tanto en situaciones agudas como crónicas (Tabla 1) [5, 6], aunque se necesitan más estudios que lo confirmen con mayor evidencia [7].

En este artículo revisamos el estado actual del tema y presentamos datos de su aplicación en la UCIP del Complejo Asistencial de Burgos.

\section{PAPEL DE LAS UNIDADES DE CUIDADOS} INTENSIVOS PEDIÁTRICOS

En las UCIP $[8,9]$ se proporciona asistencia a pacientes con patologías respiratorias diversas, tanto agudas como crónicas: problemas de vía aérea, cuadros obstructivos, enfermedades de tipo restrictivo y patología neuromuscular [10-15]. El manejo de pacientes con patología de vía aérea se realiza de forma multidisciplinar, colaborando con ORL, cirugía Infantil y unidad de trastornos respiratorios del sueño.

La VNI se aplica en situaciones agudas, para estabilizar al paciente, mientras que en casos crónicos puede ser útil durante la fase de estudio. Puede precisarse como apoyo durante el estudio fibroscópico, y para titular los parámetros de VNI en pacientes subsidiarios de soporte domiciliario (Tabla 2).

La UCIP del Hospital Universitario de Burgos es una unidad de nivel III, en la que más del $30 \%$ de los pacientes ingresan por presentar patología respiratoria o de vía aérea. Entre las medidas de soporte respiratorio, la VNI se aplica anualmente en un 44 a $50 \%$ de los casos, y supone casi el $55 \%$ de las horas de soporte (en las figuras 1 y 2 mostramos datos de 2014).

Tabla 1. Utilidad de la ventilación no invasiva (VNI).
1. Mantiene permeable vía aérea superior
2. Previene formación de atelectasias
3. Disminuye el trabajo respiratorio
4. Mejora síntomas de insuficiencia respiratoria (fre- cuencia respiratoria, disnea)
5. Disminuye hipercapnia (IPAP)
6. Evita complicaciones de intubación endotraqueal
7. Disminuye necesidades de sedación (despierto)

\section{¿CÓMO LO HACEMOS? SISTEMÁTICA DE TRABAJO}

1. ESTABILIZACIÓN DE PACIENTES CON INSUFICIENCIA RESPIRATORIA. CONSIDERACIONES SOBRE LA VNI.

En situaciones agudas lo prioritario es la estabilización del paciente. En esta fase, la VNI 
permite aliviar el trabajo respiratorio, mejorar y estabilizar la situación gasométrica y prevenir la intubación, al evitar el agotamiento. Se trata de una aplicación intensiva, en la que no podemos permitirnos periodos sin soporte. Por eso son fundamentales los clásicos requisitos para el uso de la VNI:

- Formación y motivación del equipo médico y de enfermería.

- Indicación apropiada. Adecuar equipo, interfase y programación.

- Vigilancia y cuidados. Conseguir adaptación del paciente. Evitar lesiones por presión.

- Indicación de la Técnica.

Esta técnica es eficaz (Tabla 2) tanto en patología respiratoria de tipo I (hipoxemia) como de tipo II (hipercapnia). La indicación de VNI se basa en criterios clínicos (grado de dificultad respiratoria) y gasométricos $(\mathrm{PaCO} 2>45, \mathrm{pH}$ $<7,35$, cociente $\mathrm{PaO} 2 / \mathrm{FIO} 2<250$ ), a los que recientemente se ha incorporado el uso del cociente SatO2/FiO2, aplicable cuando $\mathrm{sO} 2=$ $92 \%$ a $97 \%$. La VNI estaría indicada si el cociente $\mathrm{SpO} 2 / \mathrm{FiO} 2$ es menor de 270 en insuficiencia respiratoria tipo I (equivaldría a un $\mathrm{PaO} 2 / \mathrm{FiO} 2<300$ ), o si es menor de 235 en Insuficiencia respiratoria tipo II $(\mathrm{PaO} 2 / \mathrm{FiO} 2<$ 200) $[5,6,16]$.

Tabla 2. Patología respiratoria y VNI.

\begin{tabular}{|c|c|}
\hline Compromiso agudo & Proceso crónico \\
\hline $\begin{array}{l}\text { 19. Estabilizar vía aérea y respiración } \\
\text { - valorar uso de VNI } \\
\text { 20. Estudios } \\
\text { : Vía aérea (Fibroscopia, Imagen) } \\
\text { : Poligrafia, PSG } \\
\text { - Cardiología } \\
\text { 32. Tratamiento (multidisciplinar) } \\
\text { : Cirugía } \\
\text { - VNI } \\
\text { - Otros (Fisioterapia, nutrición, RGE...) } \\
\text { 40. Seguimiento }\end{array}$ & $\begin{array}{l}\text { 19. Estudios } \\
\text { : Via aérea (Fibroscopia, Imagen) } \\
\text { : Poligrafia, PSG } \\
\text { - Cardiología } \\
\text { 20. Tratamiento (multidisciplinar) } \\
\text { : Cirugía } \\
\text { : VNI } \\
\text { - Otros (Fisioterapia, nutrición, RGE...) } \\
\text { 40. Seguimiento }\end{array}$ \\
\hline
\end{tabular}

Para su aplicación, valoramos los factores predictores de éxito, referidos por diversos autores:

- Severidad del proceso (desfavorable).

- Cooperación y adaptación de paciente y respirador.

- $\mathrm{pH}$ (mejor si pH > 7,10).

- $\mathrm{PaCO} 2$ (mejor si PaCO2 < 90).
- Respuesta a las dos horas, con mejoría en frecuencia cardiaca, frecuencia respiratoria y gasometría.

- IRA tipo 2 (en nuestra serie hemos obtenido mejor respuesta).

- VNI como medida de soporte inicial o como apoyo a la extubación (en nuestra serie hemos obtenido mejor respuesta en este último supuesto).

En todos los casos realizamos una vigilancia inicial estrecha, procurando no retrasar la intubación si el paciente no mejora. Aunque cada vez es más amplio el abanico de aplicación de la VNI en situaciones agudas, siempre se deben considerar aquellos criterios de exclusión en los que no sería beneficiosa:

- Riesgo inminente de parada respiratoria.

- Disfunción de otros órganos (hemodinámico, neurológico...).

- Fallo en los mecanismos de protección de la vía aérea (reflejo tos, deglución...).

- Alteración facial que impida el ajuste de la interfase.

- Selección del equipo.

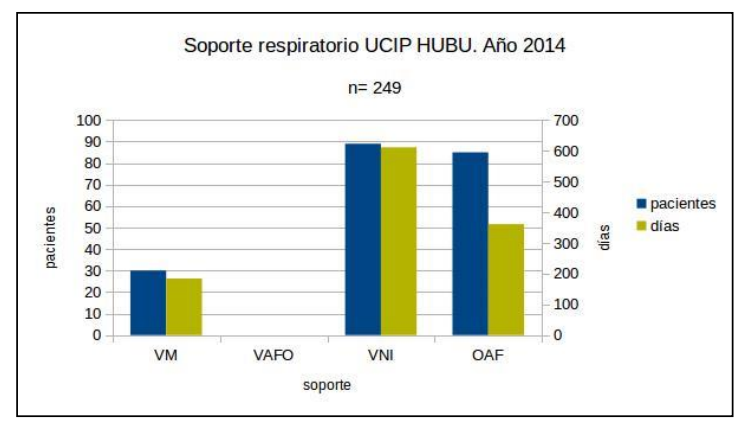

Figura 1. Soporte respiratorio. UCIP del Hospital Universitario de Burgos.

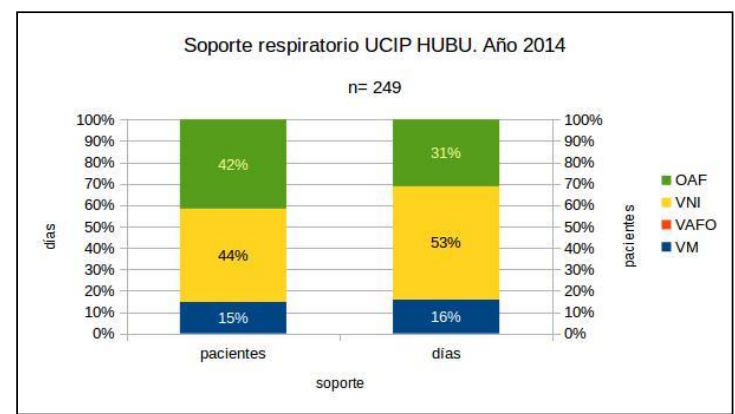

Figura 2. Soporte respiratorio. Papel de la VNI. UCIP del Hospital Universitario de Burgos. 
En cuanto a los equipos para aplicar la VNI, lo ideal es contar con aparatos específicos para $\mathrm{VNI}$ en los que se pueda regular de forma precisa la $\mathrm{FiO} 2$ aportada, puesto que la práctica totalidad de los pacientes precisan oxígeno, y un número importante necesitan $\mathrm{FiO} 2$ elevadas, al menos en las primeras horas.

En nuestro centro disponemos de una amplia gama de posibilidades:

- Respiradores de VNI de uso hospitalario (Philips V60).

- Aparatos de VNI domiciliaria.

- Respiradores de VMC dotados de función de VNI (Engstrom Carestation).

- Sistemas de flujo (infant-flow).

- Sistemas de CPAP sin ventilador («caseros», sistema Boussignac, CPAP por sistema de alto flujo, sistema helmet) (Figura $3)$.

La decisión sobre el equipo a utilizar depende de las características del paciente y de la experiencia y preferencias personales del médico (Tabla 3).

Interfase y cuidados.

La aplicación de VNI en pacientes críticos es intensiva en la mayoría de los casos, con mínimos o nulos periodos de descanso. Por eso, es fundamental la prevención de lesiones por presión, basada en la correcta fijación (interfase ajustada, no «apretada»), los cuidados de enfermería (vigilancia y cuidados de la piel, uso de apósitos en las zonas de apoyo...) y alternancia de interfases.

Los problemas de disponibilidad (pocos modelos pediátricos, sobre todo para lactantes) han sido importantes en los inicios de esta técnica en pediatría. En los últimos años, afortunadamente, está aumentando la oferta (Figura 4).

Tabla 3. Preferencia de uso de los equipos de VNI. UCIP de Burgos.

\begin{tabular}{|c|c|}
\hline Situación & Equipo \\
\hline 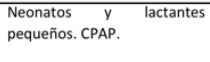 & \begin{tabular}{|ll} 
- & Ventilador de VNI de uso hospitalario (Philips V60) \\
- & Sistemas de flujo (Helmet, Infant-flow, Bubble CPAP, caseros) \\
- & Sistemas de alto flujo (Vapotherm, Fisher\&Paykel)
\end{tabular} \\
\hline 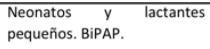 & \begin{tabular}{|l} 
- Ventilador de VNI de uso hospitalario (Philips V60) \\
- Infant-flow advanced o SiPAP
\end{tabular} \\
\hline Lactantes $y$ niños CPAP & $\begin{array}{ll}\text { - } & \text { Ventilador de VNI de uso hospitalario (Philips V60) (hipoxemia } \\
& \text { moderada-severa) } \\
\text { - } & \text { Ventilador de VNI domiciliaria (hipoxemia leve) } \\
\text { - } & \text { Sistema helmet } \\
\text { - } & \text { VMC con módulo de VNI (hipoxemia moderada-severa) } \\
- & \text { Sistema casero } \\
\text { - } & \text { Sistema Boussignac }\end{array}$ \\
\hline Lactantes y niños BiPAP & $\begin{array}{l}\text { - Ventilador de VNI de uso hospitalario (Philips V60) (hipoxemia } \\
\text { moderada-severa) } \\
\text { - Ventilador de domiciliaria (hipoxemia leve) } \\
\text { - VMC con módulo de VNI (hipoxemia moderada-severa) }\end{array}$ \\
\hline
\end{tabular}

Ediciones Universidad de Salamanca / CC BY-NC-ND

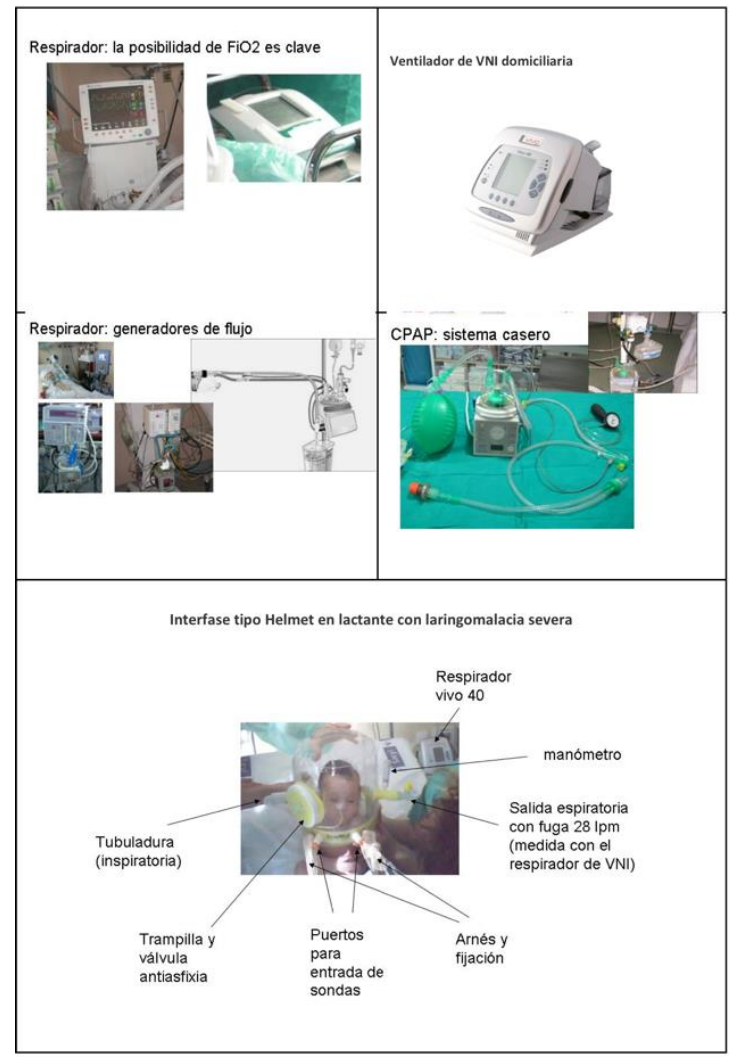

Figura 3. Distintos sistemas de VNI.
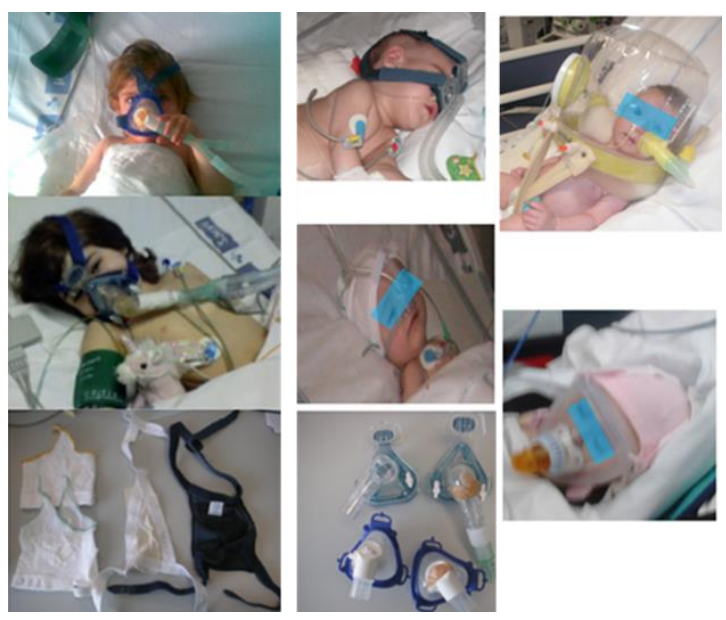

Figura 4. Interfases de VNI.

Sigue habiendo pocos datos de evidencia para recomendar de forma categórica el tipo de interfase (nasal, nasobucal, integral facial o helmet). Las recomendaciones de expertos plantean de modo muy general su elección. La calidad de materiales y equipos ha sido hasta ahora un factor que ha limitado las posibilidades. Así, mientras para el lactante pequeño y neonato, se recomendaba el uso de interfases 
nasales (al existir sistemas de contrastados resultados como el infant-flow), para el lactante mayor y niño pequeño se sugería una mayor eficacia de los sistemas nasobucales [17].

Características a tener en cuenta son:

- Eficacia.

- Confort adaptación.

- Evitar lesiones de apoyo.

- Evitar reinhalación.

- Evitar deformidades craneofaciales (VNI prolongada)

- Minimizar riesgo de sofocación.

- Uso en UCIP o en domicilio.

La experiencia acumulada nos permite sugerir que el éxito de la técnica parece depender más de la adaptación y comodidad del paciente y de la situación clínica. Las interfases faciales son las más empleadas en nuestra unidad, puesto que proporcionan una buena adaptación, evitan en general tener que alternar interfases en casos agudos, su gran superficie de apoyo reduce el riesgo de lesiones cutáneas, permiten aplicar BiPAP, admiten nebulizaciones sin interrumpir la VNI y cuentan con un buen perfil de seguridad (Figura 5). Los sistemas tipo helmet son una buena opción, sobre todo si no precisamos modo BiPAP, aunque este modo también es factible de programar.

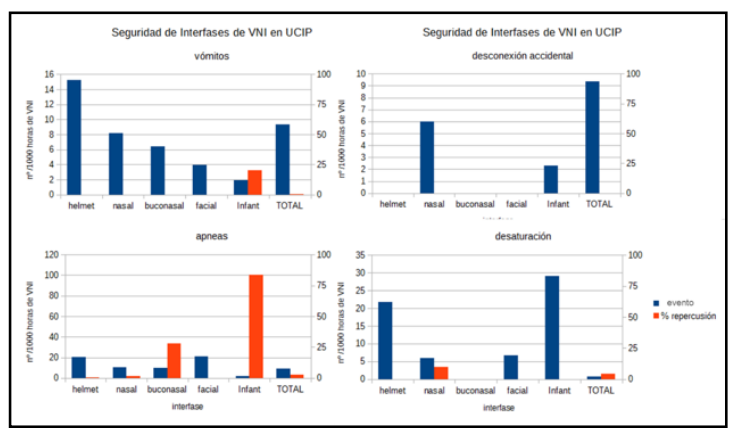

Figura 5. Seguridad de interfases empleadas en la UCIP del Hospital Universitario de Burgos.

Estas cuestiones respecto al perfil de seguridad de las interfases pudimos observarlas en un estudio a tal efecto realizado sobre datos de 44 pacientes atendidos con VNI en nuestra UCIP, en el periodo 29/03/2009 al 31/10/2014. Se recogieron datos durante $17.675,5$ horas de VNI. El $87 \%$ eran situaciones agudas y en un $57,5 \%$ había patología de base. La modalidad ventilatoria inicial más frecuente fue la CPAP (87,2\% de los casos).

Se registraron 168 vómitos, 300 desaturaciones y 292 apneas (sólo 1 precisó VPPI). Se compararon resultados entre las interfases utilizadas (helmet $6830 \mathrm{~h}, 39 \%$, nasal 5.005,5 h, $29 \%$, facial $2.542 \mathrm{~h}, 15 \%$, sistema Infant-flow $2.623 \mathrm{~h}, 15 \%$ y nasobucal $312 \mathrm{~h}, 2 \%$ ). Se observó un mayor índice de apneas por cada $1.000 \mathrm{~h}$ de VNI en las interfases helmet $(20,5)$ y buconasal (16), y datos similares en la incidencia de desaturaciones. Hubo menos desconexiones accidentales durante la ventilación con helmet y buconasal. Respecto a los riesgos atribuidos al helmet, en ninguno de los 104 vómitos registrados se ha producido obstrucción del sistema de VNI ni de la vía aérea atribuible a la interfase (Figura 5).

Programación de la VNI.

En la tabla 4 exponemos la programación inicial aplicada en nuestra unidad. Siguiendo las recomendaciones de expertos, nosotros variamos IPAP y ePAP de forma individual para lograr un adecuado reclutamiento (ePAP) y un volumen tidal y $\mathrm{PaCO} 2$ adecuados (iPAP), manteniendo la adaptación del paciente a la técnica $[5,6]$.

Tabla 4. VMNI pediátrica. Programación inicial en situaciones agudas.

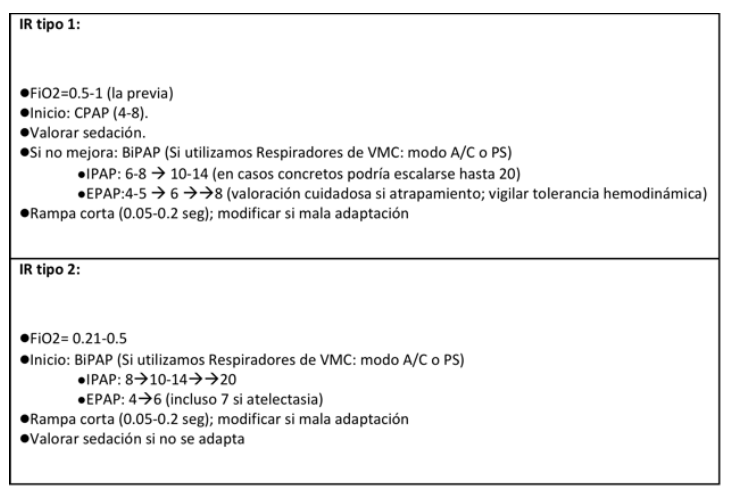

En las primeras dos horas realizamos un seguimiento muy cercano, aplicando ajustes para una mejor adaptación (sedación) y para optimizar la eficacia de la técnica (cambio de parámetros). Si no hay mejoría clínica y gasométrica consideramos que la técnica ha fracasado y nos planteamos la intubación. 
2. Estudios de PATOlogía dE VÍA AÉREA EN NIÑOS

Como venimos señalando, el planteamiento en nuestro centro se basa en la colaboración interdisciplinar (Figura 6). La UCIP facilita la realización de estudios, tanto inicial como sucesivos, mediante los que se busca:

- Titular interfase.

- Titular programación.

- Determinar tiempos de VNI.

- Determinar posibilidades para VNI en domicilio.

Para ello empleamos diversas exploraciones con las que caracterizamos la situación de la vía aérea, tanto anatómica como funcional, y la respuesta a distintos parámetros de soporte:

- Fibroscopia ORL, fibrobroncoscopia.

- Poligrafía respiratoria.

- Polisomnografía.

- Monitorización clínica, CO2 y gasometría.

- Ecocardiografía, repercusión hemodinámica.

Por tanto, es fundamental la colaboración entre diversos especialistas, jugando el ORL un papel muy importante con las exploraciones de la vía aérea.

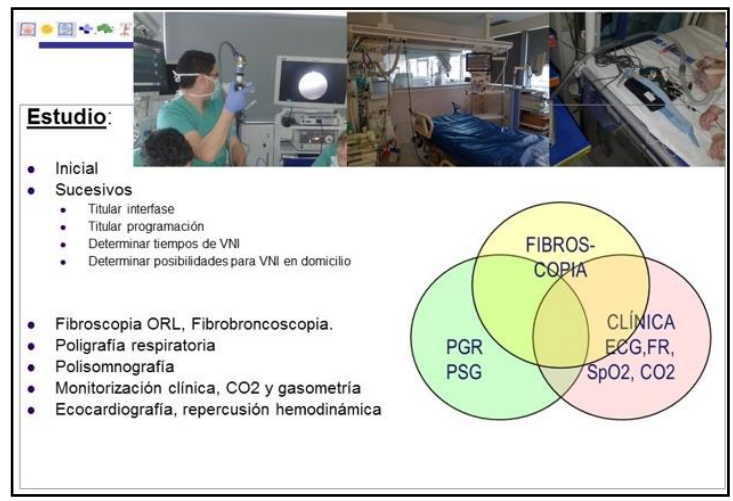

Figura 6. Estudios en patología de vía aérea.

\section{CONCLUSIONES}

La patología de vía aérea comprende un amplio espectro de manifestaciones clínicas que incluyen distintos grados de insuficiencia respiratoria y trastornos respiratorios durante el sueño.

En las UCIP se proporciona asistencia a pacientes con insuficiencia respiratoria, tanto aguda como crónica por patología de vía aérea. El manejo se realiza de forma multidisciplinar, colaborando con ORL, cirugía Infantil y unidad de trastornos respiratorios del sueño.

En situaciones agudas, se aplica VNI para estabilizar al paciente, mientras que en casos crónicos puede ser útil durante la fase de estudio. Puede precisarse como apoyo durante el estudio fibroscópico, y para titular los parámetros de VNI en pacientes subsidiarios de soporte domiciliario.

\section{BIBLIOGRAFÍA}

1. Pons M, Medina A, Esquinas A. Manual de ventilación no invasiva en pediatría. Madrid. Ergón, 2008.

2. Pons M, Gili T, Medina A, MayordomoColunga J. Ventilación no invasiva en pediatría. En: Medina Villanueva A, Pilar Orive J. Manual de ventilación mecánica pediátrica y neonatal. Capítulo 19. Ergón, Madrid 2015.

3. Balcells Ramírez J, López-Herce Cid J, Modesto Alapont V (Grupo de Respiratorio de la Sociedad Española de Cuidados Intensivos Pediátricos). Prevalencia de la ventilación mecánica en las unidades de cuidados intensivos pediátricos en España. An Pediatr (Barc). 2004;61:533-41

4. Lobete C, Medina A, Rey C, MayordomoColunga J, Concha $A$, Menéndez $S$. Correlation of SF Ratio with PF ratio in a heterogeneous sample of critically ill children. J Crit Care. 2013;28:538. e1-7.

5. Randolph AG. How are children mechanically ventilated in pediatric intensive care units? Intensive Care Med 2004;30:7467.

6. Farias JA, Frutos F, Esteban A, Casado J, Retta A, Baltodano A, et al. What is the daily practice of mechanical ventilation in pediatric intensive care units? A multicenter study. Intensive Care Med 2004;30:918-25.

7. Hamel DS, Klonin $\mathrm{H}$. The role of noninvasive ventilation for acute respiratory failure. Respir Care Clin N Am 2006;12:421-35.

8. Antonelli M, Conti G, Esquinas A, Montini L, Maggiore SM, Bello G, et al. A multiple-center survey on the use in clinical practice of noninvasive ventilation as a first-line intervention for acute respiratory distress syndrome. Crit Care Med 2007;35:18-25. 
9. Medina Villanueva A, Prieto Espuñes S, Los Arcos Solas M, Rey Galán C, Concha Torre A, Menéndez Cuervo $S$, et al. Aplicación de ventilación no invasiva en una unidad de cuidados intensivos pediátricos. An Pediatr (Barc). 2005;62(1):13-9.

10. Urbano Villaescusa J, Mencía Bartolomé S, Cidoncha Escobar E, López-Herce Cid J, Santiago Lozano MJ, Carrillo Álvarez A. Experiencia con la oxigenoterapia de alto flujo en cánulas nasales en niños. An Pediatr (Barc). 2008;68(1):4-8.

11. Raúl J. Corrales V. Ventilación mecánica no invasiva en pediatría. Consenso Chileno de Ventilación no Invasiva. Rev Chil Enf Respir 2008;24:263-5.

12. Piastra M, Antonelli M, Caresta E, Chiaretti A, Polidori G, Conti G. Noninvasive ventilation in childhood acute neuromuscular respiratory failure: a pilot study. Respiration 2006;73:791-8.
13. Nava S, Gregoretti C, Fanfulla F, Squadrone E, Grassi M, Carlucci A, et al. Noninvasive ventilation to prevent respiratory failure after extubation in high-risk patients. Crit Care Med. 2005;33:2465-70.

14. Ferrer M, Esquinas A, Arancibia F, Abuer $T$, Gonzalez G, Carrillo A, et al. Noninvasive ventilation during persisting weaning failure: a randomized controlled trial. Am J Respir Crit Care Med. 2003;168:70-6.

15. Meert AP, Berghmans $T$, Hardy $M$, Markiewicz e, Sculier JP. Noninvasive ventilation for cancer patients with life-support techniques limitation. Support Care Cancer 2006; 14:167-71.

16. Fernández-Vivas $M$, González-Díaz G, Caturla-Such J, Delgado-Vílchez FJ, Serrano-Simón JM, Carrillo-Alcaraz A, et al. Utilización de la ventilación no invasiva en la insuficiencia respiratoria aguda. Estudio multicéntrico en unidades de cuidados intensivos. Med Intensiva. 2009;33(4):15360. 